\title{
Evidence for covalent binding of epicocconone with proteins from synchronous fluorescence spectra and fluorescence lifetimes
}

\author{
DEBASHIS PANDA and ANINDYA DATTA* \\ Department of Chemistry, Indian Institute of Technology - Bombay, Powai, Mumbai 400076 \\ e-mail: anindya $a$ chem.iitb.ac.in
}

\begin{abstract}
Synchronous fluorescence and time-resolved fluorescence spectroscopic studies that reveal the interaction of epicocconone with human serum albumin is significantly different from its interaction with surfactant assemblies. This observation, along with steady-state fluorescence data, indicates groundstate interaction between the fluorophore epicocconone and the protein. Similarity in fluorescence properties with the adduct of the fluorophore with $n$-butylamine indicates that bonding occurs at the $\mathrm{N}$ terminus of the protein.
\end{abstract}

Keywords. Covalent binding; epicocconone; synchronous fluorescence spectra; fluorescence lifetimes.

\section{Introduction}

Epicocconone (figure 1), isolated from the fungus Epicoccum nigrum, is a potentially good fluorescent probe for biotechnological applications. It is characterized by high cell permeability, feeble fluorescence in neat aqueous solutions and strong fluorescence in certain media. ${ }^{1}$ This is an advantage, as it fluoresces strongly with an emission maximum at $610 \mathrm{~nm}$ upon addition of minute quantities of proteins. ${ }^{2}$ This phenomenon opens up the possibility of using epicocconone as a fluorescent sensor for the detection of proteins, which has been the subject of a significant number of fluorescence studies, using both intrinsic ${ }^{3}$ and extrinsic ${ }^{4}$ fluorescence. In this context, the study of the mode of binding of this fluorophore with proteins is of utmost importance. It is reasonable to expect the binding to be noncovalent in nature, driven by hydrophobic interaction, as the structure of epicocconone comprises a heterocyclic fused-ring system attached to a hydrocarbon tail and two polar hydroxyl groups (figure 1). Fluorophores with such structural features generally bind noncovalently with membranes and surfactant assemblies, whereby their fluorescence is enhanced considerably. ${ }^{5,6}$ In fact, such noncovalent interaction has been found to be primarily responsible for enhancement of the fluorescence of epicocconone in surfactant assemblies, due to suppression of photoisomerization, which we propose is the primary nonradiative deactivation

*For correspondence channel for this fluorophore. ${ }^{7}$ However, the fluorescence maximum in these assemblies is $520 \mathrm{~nm}$, which is the same as that in water and shows a marked blue shift as compared to the fluorescence maximum in proteins. ${ }^{2,5}$ This difference in the fluorescence maxima in membrane-like systems and proteins makes the fluorophore particularly attractive from the point of view of fluorescence imaging, as it is likely to differentiate between these two kinds of cellular components, thereby eliminating the need for its multiplexing with other fluorophores to some extent. On the other hand, it also raises the question as to why the fluorescence properties are different in these two media. A possible answer to this question is likely to be found in the recent work of Karuso and coworkers, ${ }^{2}$ where the enhancement of fluorescence of epicocconone in proteins has been proposed to be due to the covalent binding of the compound to the N-terminii of the proteins. This hypothesis is based on comparison between the steady-state fluorescence features of epicocconone in protein and those of a butylamine adduct. ${ }^{2}$ In the present paper, we address this issue by the tools of

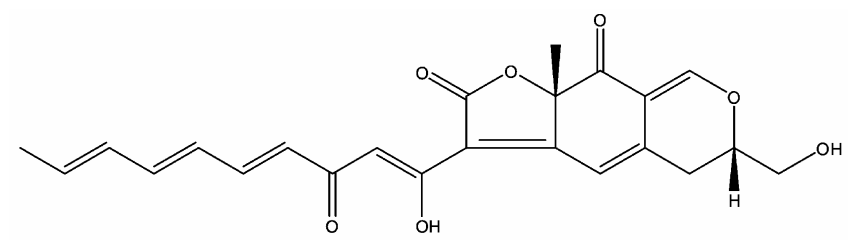

Figure 1. The molecular structure of epicocconone. 
synchronous and time-resolved fluorescence spectroscopy, performed on epicocconone, in the presence of human serum albumin (HSA), as well as on its butylamine adduct.

\section{Experimental section}

\subsection{Materials}

Deep purple total-protein stain from Amersham Biosciences, which contains epicocconone as the active ingredient, is used as received. Micromolar concentrations of the fluorophore have been maintained in all experiments. Human serum albumin (HSA), obtained from Sigma, is used as received. The surfactant sodium dodecyl sulphate (SDS) is obtained from Aldrich and used as received. $n$-Butylamine is dried with solid $\mathrm{KOH}$ and then refluxed with and distilled from $\mathrm{P}_{2} \mathrm{O}_{5}{ }^{8}$ To obtain the $n$-butylamine adduct of epicocconone, a solution of epicocconone $(10 \mu \mathrm{l} / \mathrm{ml})$ in $n$-bultylamine is stirred for $24 \mathrm{~h}$ at $4^{\circ} \mathrm{C}$. Deionized water is distilled twice before being used as the solvent. ${ }^{2}$

\subsection{Steady-state measurements}

Absorption and fluorescence spectra are recorded on a Jasco V 530 spectrophotometer and Perkin-Elmer LS55 fluorimeter, respectively. The excitation wavelength for fluorescence studies $\left(\lambda_{\mathrm{ex}}\right)$ is $430 \mathrm{~nm}$. The absorbance at this wavelength is kept below $0 \cdot 2$, in order to avoid the inner filter effects. Synchronous fluorescence studies have been performed by scanning the excitation and the emission monochromators of the fluorimeter simultaneously, with a constant offset of $20 \mathrm{~nm}$ between the two.

The steady-state anisotropy, $r_{S}$, of a system is given by

$$
r_{S}=I_{\|}-I_{\perp} / I_{\|}+2 I_{\perp}
$$

where $I_{\|}$is the polarized emission parallel to the excitation source and $I_{\perp}=G I_{\perp}^{*} \cdot \mathrm{G}$ is a correction factor for the polarization-dependence of the emission monochromator; and $I_{\perp}^{*}$ is the uncorrected intensity of emission polarized perpendicular to the excitation source. $G$ is obtained using a 'freely rotating fluorophore', coumarin 153, in methanol, where it is given by the ratio of the emission intensity of parallel and perpendicularly polarized light, i.e. $G=I_{\|} / I_{\perp}^{*}$ for a freely rotating fluorophore.

\subsection{Time-resolved measurements}

Time-resolved fluorescence measurements are performed using a picosecond pulsed-diode laser-based TCSPC fluorescence spectrometer with $\lambda_{\mathrm{ex}}=$ $406 \mathrm{~nm}$ from IBH, UK. ${ }^{7}$ Emission from the samples at $620 \mathrm{~nm}$ is collected at right angles to the direction of the excitation beam, at magic angle polarization, except for the anisotropy measurements. The full width at half maximum of the instrument-response function is $250 \mathrm{ps}$ and the resolution is $7 \mathrm{ps}$ per channel. The data are fitted to multiexponential functions after deconvolution of the instrument response function by an iterative reconvolution technique, using the IBH DAS 6.0 data analysis software, where reduced $\chi^{2}$ and weighted residuals serve as parameters for goodness of fit. ${ }^{9}$

Time-dependent anisotropy, $r(t)$, is constructed from the decays in the parallel and perpendicular directions to that of the excitation polarization $\left(I_{\|}(t)\right.$ and $I_{\perp}(t)$ respectively) as:

$$
r(t)=I_{\|}(t)-G I_{\perp}(t) / I_{\|}(t)+2 G I_{\perp}(t) .
$$

The $G$ factor is calculated from tail matching of the decays. The decays of $r(t)$ are fitted to single and multiexponential functions as required, using the formula

$$
r(t)=r_{0} \sum_{i} a_{i} \exp \left(-t /\left(\tau_{r}\right)_{i}\right) .
$$

\section{Results and discussion}

\subsection{Steady-state fluorescence of epicocconone in protein}

The absorption spectra of epicocconone in HSA and $n$-butylamine exhibit maxima at $530 \mathrm{~nm}$ and $546 \mathrm{~nm}$ respectively (table 1 , figure 2 ). These shows considerably red shift compared to the absorption maximum at $437 \mathrm{~nm}$ in neat aqueous solution. Earlier, we have observed a remarkable red shift to $560 \mathrm{~nm}$ in the absorption maximum of epicocconone in cetyl trimethyl ammonium bromide (CTAB) micelle. This has been explained by the deprotonation of enolic proton in the Stern layer of the micelle, which has a 
considerably higher $\mathrm{pH}$ than neat water. ${ }^{7,10}$ Since the local $\mathrm{pH}$ of the protein solution is not significantly altered, we infer the presence ground-state interaction of the fluorophore with the protein, as no spectral shift has been observed in the neutral triton $\mathrm{X}$ 100 or anionic SDS micelle, indicating that the shift observed in the presence of HSA cannot be merely due to a change in the microenvironment of the fluorophore. The fluorescence maximum in HSA is at $610 \mathrm{~nm}$ and the fluorescence quantum yield is 0.06 , which is seven times more than the value in neat aqueous solution. Upon addition of $1 \mathrm{mM}$ SDS to the solution containing epicocconone and HSA, a further twofold enhancement of fluorescence is observed and the maximum shifts to $602 \mathrm{~nm}$. Further addition of SDS (up to $3 \mathrm{mM}$ ) leads to enhancement of quantum yield, while at $10 \mathrm{mM}$ SDS the quantum yield, decreases. This observation indicates that the fluorophore experiences a greater hydrophobic environment in the HSA-SDS aggregate, as compared to that in native HSA, which is in agreement with the earlier observation of comparatively apolar microenvironment of the protein-surfactant aggregates,

Table 1. Fluorescence maxima and quantum yield of epicocconone in different media.

\begin{tabular}{lccc}
\hline & $\lambda_{\text {abs }}^{\max }(\mathrm{nm})$ & $\lambda_{\mathrm{em}}^{\max }(\mathrm{nm})$ & $\phi_{f}$ \\
\hline $\mathrm{H}_{2} \mathrm{O}$ & 437 & 520 & $0 \cdot 008$ \\
$\mathrm{HSA}$ & 530 & 610 & $0 \cdot 06$ \\
$\mathrm{HSA}+1 \mathrm{mM}$ SDS & 525 & 602 & $0 \cdot 11$ \\
$\mathrm{HSA}+3 \mathrm{mM}$ SDS & 525 & 600 & $0 \cdot 14$ \\
$\mathrm{HSA}+5 \mathrm{mM} \mathrm{SDS}$ & 522 & 599 & $0 \cdot 14$ \\
$\mathrm{HSA}+10 \mathrm{mM}$ SDS & 520 & 599 & $0 \cdot 13$ \\
$n$-Butylamine & 545 & 579 & - \\
\hline
\end{tabular}

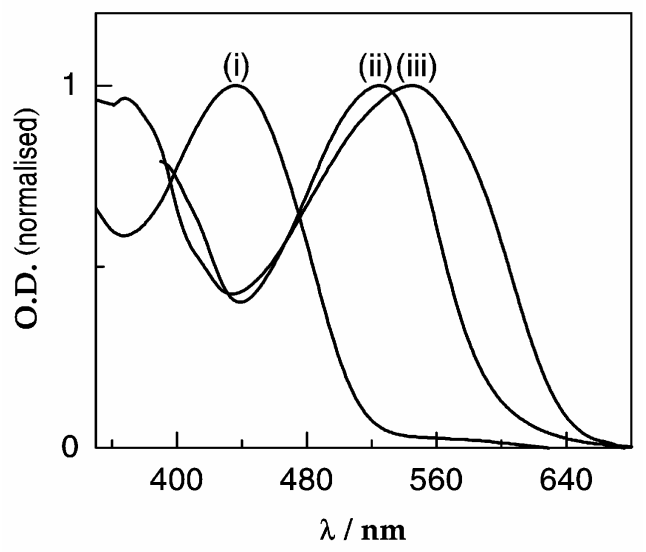

Figure 2. Absorption spectra of epicocconone in (i) $\mathrm{H}_{2} \mathrm{O}$ (ii) $\mathrm{HSA}\left(1 \mathrm{mg} \mathrm{mL}^{-1}\right)+\operatorname{SDS}(1 \mathrm{mM})$ and (iii) $n$ butylamine. due to the lesser availability of water molecules. ${ }^{10}$ Of course, in a complicated system like this, it is possible that the fluorophore might be distributed in different microdomains and so, it is imperative to investigate the ground-state heterogeneity of the fluorophore, which is done by synchronous fluorescence spectroscopy.

\subsection{Synchronous fluorescence experiments}

The synchronous fluorescence technique is a convenient tool to study ground state heterogeneity of fluorophores. ${ }^{11,12}$ In this experiment, excitation and emission wavelengths are varied simultaneously with a constant offset, chosen by trial and error, such that the experiment is performed along the diagonal of the excitation-emission matrix, which passes through the maximum number of peaks and troughs. The resulting spectra have a greater number of narrower peaks compared with that obtained in conventional excitation spectroscopy, allowing dis-

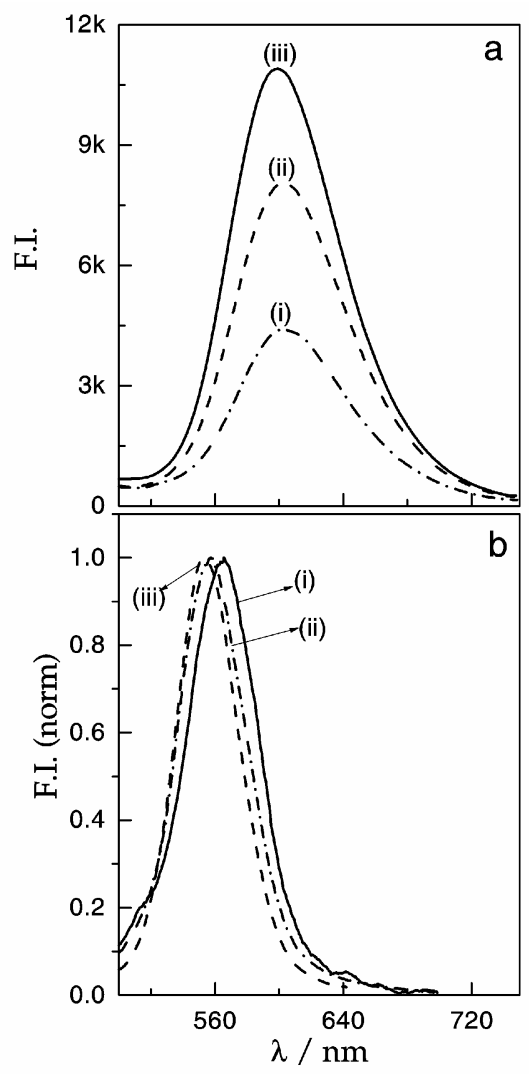

Figure 3. (a) Conventional fluorescence spectra and (b) synchronous fluorescence spectra of epicocconone in (i) HSA $\left(1 \mathrm{mg} \mathrm{mL}^{-1}\right)$, and (ii) HSA $\left(1 \mathrm{mg} \mathrm{mL}^{-1}\right)+\mathrm{SDS}$ (1 mM), and (iii) HSA (1 $\left.\mathrm{mg} \mathrm{mL}^{-1}\right)+\operatorname{SDS}(3 \mathrm{mM})$. 
crimination between fluorophores that have broad and overlapping spectra. In the present experiment, the synchronous fluorescence spectrum of epicocconone in protein exhibits a single peak at $566 \mathrm{~nm}$ and experiences a blue shift of $10 \mathrm{~nm}$ upon addition of SDS (figure $3 \mathrm{~b}$ ), whereas in all the three micelles, the peak is at $480 \mathrm{~nm}$ (figure 4). Further addition of SDS causes blue shift the peak into $552 \mathrm{~nm}$ (figure $3 b)$. This indicates the presence of a single groundstate species that is emissive in these media, which is significantly different from the emissive species in micelles. Interestingly, the synchronous fluorescence spectrum of epicocconone in bultyamine exhibits a peak at $556 \mathrm{~nm}$, which is identical to the spectral maximum in the HSA-SDS aggregate, indicating that the mode of interaction of fluorophore with protein is similar to that $n$-butylamine and is different from the noncovalent interaction with micelles. This is in agreement with the original hypothesis of Karuso and coworkers. ${ }^{2}$ The dip in synchronous fluorescence spectra of epicocconone in $n$-butylamine could be due to the presence of undesired product obtained from reaction of epicocconone with $n$-butylamine. To further substantiate this contention, we have performed time resolved fluorescence studies, which have been discussed in the next section.

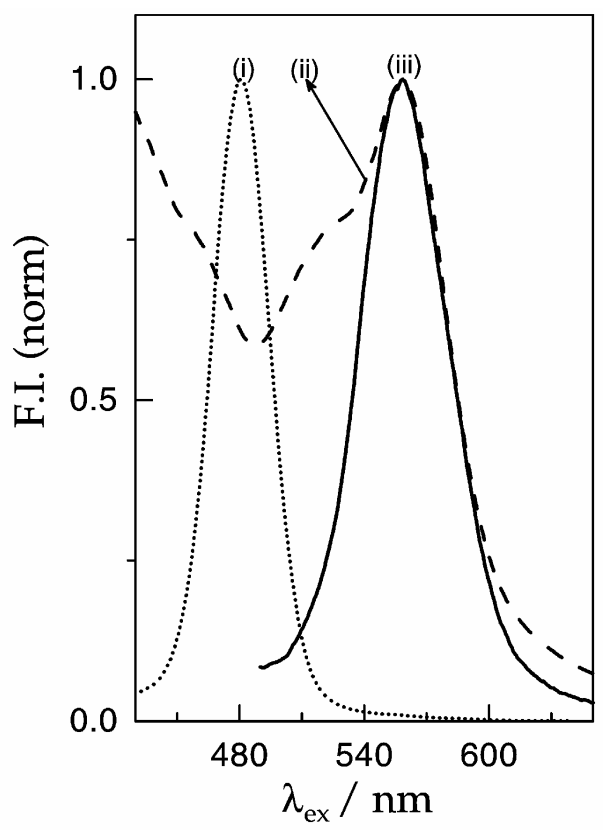

Figure 4. The synchronous fluorescence spectra of epicocconone in (i) TX $100(1 \mathrm{mM})$, (ii) $n$-butylamine, and (iii) $\operatorname{HSA}\left(1 \mathrm{mg} \mathrm{mL}^{-1}\right)+\operatorname{SDS}(1 \mathrm{mM})$.

\subsection{Time-resolved fluorescence experiments}

Fluorescence anisotropy decay parameters at $620 \mathrm{~nm}$ are tabulated in table 2 . The value of limiting anisotropy, $r(0)$, obtained in the time-dependent measurement is quite small, even though the steady-state anisotropy is quite significant, indicating an ultrafast component that is missed due to the limited time resolution of our experiment. This component is likely to be due to conformational changes of the conjugated aliphatic chain of the molecule. However, relaxation times of $5.63 \mathrm{~ns}$ and $8.54 \mathrm{~ns}$ are obtained in HSA and HSA-SDS complexes, respectively (table 2). These are similar to the time constants associated with the tumbling motion of the proteins and thus, serve as an evidence of incorporation of the fluorophore in the protein. ${ }^{13}$ SDS at lower concentration causes a hydrophobic collapse of the protein, leading to the blue shift of fluorescence maxima. The nanosecond depolarisation time has contributions from the rotational motion of the fluorophore itself and the tumbling motion of the protein. A more rigid microenvironment around the fluorophore is expected to increase the rotational relaxation time, which is observed. However, the values of $r_{0}$ observed are really very small, indicating that there is an ultrafast component of the depolarisation process, which is likely to be associated with the photoprocess itself. This component is missed due to the limitation of the time resolution of our setup. Steady-state anisotropy, which has contributions from both the observed nanosecond component and this ultrafast component, remains almost constant.

Fluorescence decays of epicocconone in HSA (figure 5) are bimodal with lifetimes of $0.91 \mathrm{~ns}$ and $2 \cdot 12 \mathrm{~ns}$, which are significantly longer than the time constants of $0.25 \mathrm{~ns}$ and $1.13 \mathrm{~ns}$ observed in micelles (table 3). On addition of $1 \mathrm{mM}$ SDS to HSA the lifetime of the short component is increased to $1.28 \mathrm{~ns}$ and of the longer one to $2.69 \mathrm{~ns}$. At $3 \mathrm{mM}$ SDS concentration the lifetime of the short component is further increased to $1.84 \mathrm{~ns}$ and that of the longer one to $3.04 \mathrm{~ns}$, whereas at $10 \mathrm{mM}$ SDS both get

Table 2. Time-resolved anisotropy parameters.

\begin{tabular}{lcccc}
\hline & $r_{S}$ & $r_{0}$ & $\tau_{r}(\mathrm{~ns})$ & $\chi^{2}$ \\
\hline HSA & 0.31 & 0.06 & 5.63 & 1.02 \\
HSA + 1 mM SDS & 0.30 & 0.03 & 8.54 & 1.00 \\
\hline
\end{tabular}


Table 3. Time-resolved fluorescence parameters of epicocconone in different media.

\begin{tabular}{lccccccc}
\hline & $\tau_{1}(\mathrm{~ns})$ & $\tau_{2}(\mathrm{~ns})$ & $\tau_{3}(\mathrm{~ns})$ & $a_{1}$ & $a_{2}$ & $a_{3}$ & $\chi^{2}$ \\
\hline $\mathrm{H}_{2} \mathrm{O}$ & 0.25 & 1.13 & & 0.80 & 0.20 & & 1.13 \\
$\mathrm{HSA}$ & 0.91 & 2.12 & & 0.54 & 0.46 & 1.02 \\
$\mathrm{HSA}+1 \mathrm{mM} \mathrm{SDS}$ & 1.28 & 2.69 & & 0.34 & 0.66 & & 1.08 \\
$\mathrm{HSA}+3 \mathrm{mM} \mathrm{SDS}$ & 1.84 & 3.04 & & 0.60 & 0.40 & & 1.02 \\
$\mathrm{HSA}+5 \mathrm{mM} S D S$ & 1.80 & 2.98 & & 0.59 & 0.41 & & 1.06 \\
$\mathrm{HSA}+10 \mathrm{mM} \mathrm{SDS}$ & 1.22 & 2.43 & & 0.41 & 0.59 & & 1.14 \\
$n$-Butylamine & 0.86 & 3.55 & 0.14 & 0.20 & 0.07 & 0.73 & 1.00 \\
\hline
\end{tabular}

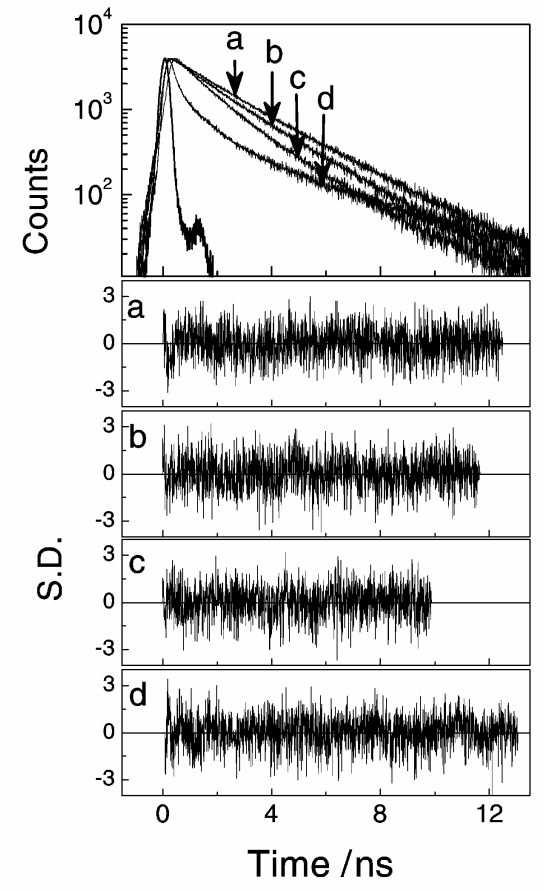

Figure 5. Fluorescence decay profiles of epicocconone in (a) $\mathrm{HSA}\left(1 \mathrm{mg} \mathrm{mL}^{-1}\right)+\mathrm{SDS}(3 \mathrm{mM})$, (b) HSA $\left(1 \mathrm{mg} \mathrm{mL} L^{-1}\right)+\operatorname{SDS}(1 \mathrm{mM})$, (c) $\mathrm{HSA}\left(1 \mathrm{mg} \mathrm{mL}^{-1}\right)$ and (d) $n$-butylamine.

quenched to $1.22 \mathrm{~ns}$ and $2.43 \mathrm{~ns}$ respectively. In HSA, the relative abundances are almost equal while on addition of $3 \mathrm{mM}$ surfactant SDS, the relative abundance of the short component becomes 0.60 and that of the long component $0 \cdot 40$. This observation is commensurate with our recent results on 2-(2'pyridyl)benzimidazole in protein-surfactant aggregates, where upon addition of SDS, the $60 \mathrm{ps}$ component in water increases in lifetime to values of $130 \mathrm{ps}$ in case of BSA and $80 \mathrm{ps}$ in case of HSA. ${ }^{14}$ At $10 \mathrm{mM}$ SDS concentration the relative abundances get reversed: 0.41 for the short and 0.59 for the long component. The study on HSA-SDS interactions revealed that there are mainly three species (native HSA, HSA-SDS complex, and partially de- natured HSA) in solution depending upon the concentration of SDS. ${ }^{15}$ At lower concentrations of SDS $(<0.3 \mathrm{mM})$, equilibrium exists between three species while at concentrations greater than $0.5 \mathrm{mM}$ it gets restricted between two species (HSA-SDS complex, and partially denatured HSA). At SDS concentration $(>8 \mathrm{mM})$ only partially denatured HSA exists in solution. ${ }^{15}$ In our case, quenching of epicocconone in protein solution at higher concentration of SDS indicates that SDS-induced structural change of HSA is able to alter the dynamics of fluorophores. However, fluorescence decays of epicocconone in $n$-butylamine exhibit the presence of three components: $0.14 \mathrm{~ns}, 0.86 \mathrm{~ns}$ and $3.55 \mathrm{~ns}$. Except the ultrafast component in $n$-butylamine, the other two lifetimes of epicocconone are quite close to the lifetimes observed in protein and protein-surfactant systems. So, we conclude that epicocconone binds covalently to the N-terminus of the proteins and the adduct is similar in nature to its adduct with butylamine. ${ }^{2}$ The presence of the $0 \cdot 14 \mathrm{~ns}$ component in butylamine and absence thereof in the protein could be due to the presence of quencher in $n$-butylamine.

\section{Conclusion}

The nature of interaction of epicocconone with protein is significantly different from its interaction with surfactant assemblies. The $\mathrm{N}$-terminus of protein interacts with epicocconone and forms a stable covalent complex. This is quite evident from the interaction of epicconone with $n$-butylamine. The equilibrium constant and hence, the $\Delta G^{0}$ of the interaction of epicocconone with HSA calculated from the double reciprocal plot of change in fluorescence intensity and concentration of the protein are $3.02 \times 10^{6} \mathrm{M}^{-1}$ and $-8.83 \mathrm{kcal} \mathrm{mol}^{-1}$ respectively. These values indicate strong binding of the fluorophore to the protein $^{16}$ and support the contention of a covalent binding. The enhancement of quantum yield and 
fluorescence lifetime of epicocconone on addition of SDS to HSA indicates the facilitation of the mode of interaction of fluorophore with protein. These inferences are bolstered by the steady-state data and the temporal features. In summary, the present study supports the mechanism of interaction of epicocconone with protein by Karuso and coworkers. ${ }^{2}$

\section{Acknowledgement}

This work is supported by a Council of Scientific and Industrial Research (CSIR) research grant. DP thanks the CSIR for a fellowship.

\section{References}

1. Bell P J L and Karuso P 2003 J. Am. Chem. Soc. 125 9304

2. Coghlan, D R, Mackintosh J A and Karuso P 2005 Org. Lett. 72401

3. Swaminathan R, Krishnamoorthy G and Periasamy N 1994 Biophys. J. 672013
4. Sytnik A and Litvinyuk I 1996 Proc. Natl. Acad. Sci. USA 9312959

5. Goodwin A P, Mynar J L, Ma Y, Fleming G R and Frechet J M J 2005 J. Am. Chem. Soc. 1279952

6. Badugu R and Sakamoto K 2003 Chem. Commun. 1368

7. Panda D, Khatua S and Datta A 2006 J. Phys. Chem. B111 1648

8. Armarego W L F and Perrin D D 1996 Purification of laboratory chemicals 4th edn (Oxford: ButterworthHeinemann) p. 125

9. Panda D and Datta A 2006 J. Chem. Phys. 125 054513

10. Sahu K, Roy D, Mondal S K, Karmakar R and Bhattacharyya K 2005 Chem. Phys. Lett. 404341

11. Patra D and Mishra A K 2000 Analyst 1251383

12. Askari M D F and Vo-Dinh T 2004 Biopolymers 73 510

13. Rami B R, Krishnamoorthy G and Udgaonkar J B 2003 Biochemistry 427986

14. Mukherjee T K, Lahiri P and Datta A 2006 (in press) (doi: 10.1016/j.cplett.2007.03.014)

15. Gelamo E L and Tabak M 2000 Spectrochim. Acta A56 2255

16. Athar H, Ahmad, N, Tayyab S and Qasim M A 1999 Int. J. Biol. Macromol. 25353 